\title{
Bound state spectra of three-body muonic molecular ions
}

\author{
Alexei M. Frolov* and David M. Wardlant \\ Department of Chemistry \\ University of Western Ontario, \\ London, Ontario N6H 5B\%, Canada
}

(Dated: May 29, 2018)

\begin{abstract}
The results of highly accurate calculations are presented for all twenty-two known bound $S(L=$ $0)-, P(L=1)-, D(L=2)-$ and $F(L=3)$-states in the six three-body muonic molecular ions $p p \mu, p d \mu, p t \mu, d d \mu, d t \mu$ and $t t \mu$. A number of bound state properties of these muonic molecular ions have been determined numerically to high accuracy. The dependence of the total energies of these muonic molecules upon particle masses is considered. We also discuss the current status of muon-catalysis of nuclear fusion reactions.
\end{abstract}

PACS number(s): 36.10.+Di, 36.10.-k and 31.10.+z.

${ }^{*} \mathrm{E}$-mail address: afrolov@uwo.ca

${ }^{\dagger} \mathrm{E}$-mail address: dwardlaw@uwo.ca 


\section{INTRODUCTION}

In this study we consider the bound state spectra of the muonic molecular ions $p p \mu, p d \mu, p t \mu, d d \mu, d t \mu$ and $t t \mu$. In this paper the notations $p, d, t$ designate the nuclei of hydrogen isotopes (protium, deuterium and tritium, respectively), while $\mu$ means the negatively charged muon $\mu^{-}$. Our main goal is to determine total energies and other bound state properties in these muonic molecular ions to high enough numerical accuracy to be sufficient for all current and anticipated future experimental needs.

In general, the bound state spectra in the six muonic molecular ions $p p \mu, p d \mu, p t \mu$, $d d \mu, d t \mu$ and $t t \mu$ can be separated into three different groups [2] on qualitative grounds. The first group includes the three light muonic molecular ions $p p \mu, p d \mu$ and $p t \mu$. Each of these systems has two bound states: one $S(L=0)$-state and one $P(L=1)$-state, where the notation $L$ means the total angular momentum of the three-body system. Neither of these two states is weakly bound. Note that each of these light muonic molecular ions contains at least one protium nucleus. The second group includes the two 'intermediate' muonic molecular ions $d d \mu$ and $d t \mu$ each of which has five bound states: two $S(L=0)$-states, two $P(L=1)$-states and one $D(L=2)$-state. One of these five states (the excited $P^{*}(L=1)$-state) in each of these ions is weakly bound. The third group contains only the heaviest muonic molecular ion $t t \mu$ which has six bound states (and no weakly bound states): two $S(L=0)$-states, two $P(L=1)$-states, one $D(L=2)$-state and one $F(L=3)$-state.

It can be shown (see, e.g., [3] ) that the total number of bound states in any muonic molecular ion $a^{+} b^{+} \mu^{-}$is determined by the lightest nucleus in this ion. This explains why only three groups of different bound state spectra can be found in six such ions. Moreover, it follows that there must be a similarity between the energy spectra of the 'protium' muonic molecular ions $p p \mu, p d \mu$ and $p t \mu$. An analogous similarity can be found in the bound state spectra of the $d d \mu$ and $d t \mu$ ions. It can be shown that in such 'families' of muonic molecular ions the symmetric ion, e.g., $p p \mu$, always has the maximal binding energy of the three protium ions: $p p \mu, p d \mu$ and $p t \mu$. By using these similarities between the bound state spectra in each of these 'families', one also finds a number of useful relations for the total and binding energies as well as for other bound state properties of different muonic molecular ions. For instance, let us assume that we know that the excited $P$-state in the $d d \mu$ ion is weakly bound and its binding energy is $\approx-1.9745 \mathrm{eV}$ (see, e.g., [2]). From the similarity of the 
bound state spectra of the $d d \mu$ and $d t \mu$ ions it one predicts that the corresponding excited $P$-state in the $d t \mu$ ion is also weakly bound and its binding energy is above $-1.9745 \mathrm{eV}$, i.e. $-1.9745 \mathrm{eV} \leq \varepsilon\left(d t \mu, P^{*}(L=1)\right)<0$. This prediction turns out to be correct, as the $d t \mu$ ion has a weakly bound excited $P$-state with the binding energy $\approx-0.660 \mathrm{eV}$.

Our labels for the bound states in muonic molecular ions are based on atomic $L S$ notations (see, e.g., [4]). Note that there is another classification scheme which is still in use for muonic molecular ions and which was originally introduced to classify bound state spectra in adiabatic molecular ions, e.g., in the $\mathrm{H}_{2}^{+}$molecular ion [5]. In this scheme each bound state is designated by its rotational $J$ and vibrational $\nu$ quantum numbers, i.e., we have the $(J, \nu)$-states. The ground state in any muonic molecular ion is designated as the (0,0)-state, while the excited $P$-state in this scheme is denoted as the $(1,1)$-state, etc. Each of these classification schemes has its own advantages and disadvantages in applications to actual systems. Note also that there is a uniform correspondence between the 'atomic' and 'molecular' classification schemes.

\section{THE HAMILTONIAN AND WAVE FUNCTIONS}

As mentioned above in this study we consider the bound state spectra in the six muonic molecular ions $p p \mu, p d \mu, p t \mu, d d \mu, d t \mu$ and $t t \mu$. All particles which form such three-body ions are assumed to be point and structureless. Each of these three particles has a finite mass which equals one of the masses $m_{\mu}, m_{p}, m_{d}$ and/or $m_{t}$; the electric charges are $q_{\mu}=-1$ and $q_{p}=q_{d}=q_{t}=+1$ (in muon-atomic units, where $\hbar=1, m_{\mu}=1, e=1$ ). The Hamiltonian $H$ of the three-body muonic molecular ions is written in the form, e.g., for the $a^{+} b^{+} \mu^{-}$ion

$$
H=-\frac{\hbar^{2}}{2 m_{\mu}}\left(\frac{m_{\mu}}{m_{a}} \nabla_{a}^{2}+\frac{m_{\mu}}{m_{b}} \nabla_{b}^{2}+\nabla_{\mu}^{2}\right)+\frac{q_{a} q_{b}}{r_{a b}}+\frac{q_{a} q_{\mu}}{r_{a \mu}}+\frac{q_{b} q_{\mu}}{r_{b \mu}}
$$

where $\nabla_{i}=\left(\frac{\partial}{\partial x_{i}}, \frac{\partial}{\partial y_{i}}, \frac{\partial}{\partial z_{i}}\right)$ and $i=a, b, \mu$. In muon-atomic units we have

$$
H=-\frac{1}{2}\left(\frac{1}{m_{a}} \nabla_{a}^{2}+\frac{1}{m_{b}} \nabla_{b}^{2}+\nabla_{\mu}^{2}\right)+\frac{1}{r_{a b}}-\frac{1}{r_{a \mu}}-\frac{1}{r_{b \mu}}
$$

where the two masses $m_{a}$ and $m_{b}$ of nuclei of two hydrogen isotopes must be expressed in terms of the muon mass $m_{\mu}$. In fact, in this study only the muon-atomic units $\left(\hbar=1, m_{\mu}=\right.$ $1, e=1)$ are used. Advantages of these units are discussed in Section IV below.

Our computational goal is to determine exceptionally accurate solutions, i.e., the eigenstates and corresponding wave functions of the non-relativistic Schrödinger equation 
$H \Psi\left(\mathbf{r}_{1}, \mathbf{r}_{2}, \mathbf{r}_{3}\right)=E \cdot \Psi\left(\mathbf{r}_{1}, \mathbf{r}_{2}, \mathbf{r}_{3}\right)$, where $E<0$ and the non-relativistic Hamiltonian is written in the form of Eq.(2). In actual calculations the wave functions of muonic molecular ions are usually approximated with the use of different variational expansions. In this work we shall consider the exponential variational expansion in relative coordinates $r_{12}, r_{13}, r_{23}$ [6]. Here and everywhere below in this work the notation $r_{i j}=\left|\mathbf{r}_{i}-\mathbf{r}_{j}\right|=r_{j i}$ designates the relative coordinate between particles $i$ and $j$. In many cases, however, it is very convenient to introduce three new variables $u_{1}, u_{2}, u_{3}$ which are called perimetric coordinates. They are simply related to the three relative coordinates: $u_{i}=\frac{1}{2}\left(r_{i k}+r_{i j}-r_{j k}\right)$, and therefore, $r_{i j}=u_{i}+u_{j}$, where $(i, j, k)=(1,2,3)$. The perimetric coordinates are truly independent, and each of them varies from 0 to $+\infty$. This significantly simplifies derivation of the explicit formulas for all matrix elements needed in highly accurate computations of the bound states. The explicit form of the exponential variational expansion in perimetric/relative coordinates is

$$
\begin{array}{r}
\Psi_{L M}=\frac{1}{2}\left(1+\kappa \hat{P}_{21}\right) \sum_{i=1}^{N} \sum_{\ell_{1}} C_{i} \mathcal{Y}_{L M}^{\ell_{1}, \ell_{2}}\left(\mathbf{r}_{31}, \mathbf{r}_{32}\right) \phi_{i}\left(r_{32}, r_{31}, r_{21}\right) \\
\exp \left(-\alpha_{i} u_{1}-\beta_{i} u_{2}-\gamma_{i} u_{3}\right) \times \\
\exp \left(\imath \delta_{i} u_{1}+\imath e_{i} u_{2}+\imath f_{i} u_{3}\right)
\end{array}
$$

where $C_{i}$ are the linear (or variational) parameters, $\alpha_{i}, \beta_{i}, \gamma_{i}, \delta_{i}, e_{i}$ and $f_{i}$ are the non-linear parameters and $\imath$ is the imaginary unit.

The functions $\mathcal{Y}_{L M}^{\ell_{1}, \ell_{2}}\left(\mathbf{r}_{31}, \mathbf{r}_{32}\right)$ in Eq.(3) are the bipolar harmonics [7] of the two vectors $\mathbf{r}_{31}=r_{31} \cdot \mathbf{n}_{31}$ and $\mathbf{r}_{32}=r_{32} \cdot \mathbf{n}_{32}$. The bipolar harmonics are defined as follows [7]

$$
\mathcal{Y}_{L M}^{\ell_{1}, \ell_{2}}(\mathbf{x}, \mathbf{y})=x^{\ell_{1}} y^{\ell_{2}} \sum_{\ell_{1}, \ell_{2}} C_{\ell_{1} m_{1} ; \ell_{2} m_{2}}^{L M} Y_{\ell_{1} m_{1}}\left(\mathbf{n}_{x}\right) Y_{\ell_{2} m_{2}}\left(\mathbf{n}_{y}\right)
$$

where $C_{\ell_{1} m_{1} ; \ell_{2} m_{2}}^{L M}$ are the Clebsch-Gordan coefficients (see, e.g., [7]) and the vectors $\mathbf{n}_{x}=\frac{\mathbf{x}}{x}$ and $\mathbf{n}_{y}=\frac{\mathbf{y}}{y}$ are the corresponding unit vectors constructed for arbitrary non-zero vectors $\mathbf{x}$ and $\mathbf{y}$. Also, in this equation $L$ is the total angular momentum of the three-body system, i.e. $\hat{L}^{2} \Psi_{L M}=L(L+1) \Psi_{L M}$, while $M$ is the eigenvalue of the $\hat{L}_{z}$ operator, i.e. $\hat{L}_{z} \Psi_{L M}=$ $M \Psi_{L M}$. In actual calculations it is possible to use only those bipolar harmonics for which $\ell_{1}+\ell_{2}=L+\epsilon$, where $\epsilon=0$ or 1 . The first choice of $\epsilon$ (i.e. $\epsilon=0$ ) corresponds to the natural spatial parity $\chi_{P}=(-1)^{L}$ of the wave functions. The second choice (i.e. $\epsilon=1$ ) represents states with the unnatural spatial parity $\chi_{P}=(-1)^{L+1}$. In this work we shall consider only the bound states of natural parity, since only such states exist in real physical systems. An additional family of polynomial-type functions $\phi_{i}\left(r_{32}, r_{31}, r_{21}\right)$ are also used in 
Eq.(3) to represent the inter-particle correlations at short distances. In general, these simple polynomial functions allow one to increase the overall flexibility of the variational expansion Eq.(3). In our present calculations, however, these additional functions were chosen in the form $\phi_{i}\left(r_{32}, r_{31}, r_{21}\right)=1$ for $i=1, \ldots, N$. The operator $\hat{P}_{21}$ in Eq.(3) is the permutation of the identical particles in symmetric three-body systems, where $\kappa= \pm 1$, otherwise $\kappa=0$. In this study we assume that $\kappa=\chi_{P}=(-1)^{L}$ for all symmetric muonic molecular ions $p p \mu, d d \mu, t t \mu$ and $\kappa=0$ for all non-symmetric ions $p d \mu, p t \mu, d t \mu$.

In general, highly accurate computations of bound states in muonic molecular ions are not easy to perform, since there are bound states with different angular momenta $L$ ( $L$ $=0,1,2$ and 3 ) and some of these states are very weakly bound. Variational expansions used in highly accurate calculations must provide fast convergence rates for each of the bound states, including all weakly bound states. Note that the actual goal of many current calculations of muonic molecular ions is the computation of various bound state properties, rather than the energies. In general, the convergence rate for some of these properties, including many nuclear-nuclear expectation values, e.g., the expectation values which include the nuclear-nuclear delta-function $\delta_{++}$(e.g., the $\left\langle\delta_{++} \frac{\partial^{n}}{\partial r_{++}^{n}}\right\rangle$ expectation values for $n \geq 1$ ), is substantially slower than for the total energies. This explains our current need for highly accurate wave functions. For instance, as follows from numerical calculations to determine the $\left\langle\delta_{++} \frac{\partial^{n}}{\partial r_{++}^{n}}\right\rangle$ expectation values, to the accuracy $\pm 1 \cdot 10^{-8}$ one needs to use wave functions which provide an accuracy $\approx 1 \cdot 10^{-15} a . u$. for the total energy. Such values are needed in computations of the lowest order relativistic and QED corrections. A separate, but serious problem for accurate computations of three-body systems is the adiabatic divergence described in [8]. This problem always appears when 'pure atomic' variational expansions are applied to the two-center Coulomb systems and/or to the systems close to them. For our systems this means that the convergence rates observed for the $p p \mu$ and $p d \mu$ ions are relatively high in comparison to the analogous convergence rates for the $d t \mu$ and $t t \mu$ ions which are substantially slower.

Nevertheless, variational calculations of bound states in muonic molecular ions are of great interest for predicting the physics of few-body systems as well as in some applications. In general, the study of bound state spectra in muonic molecular ions has provided us with a large amount of very valuable information and drastically improved our knowledge about the bound state spectra in arbitrary Coulomb three-body systems. Furthermore, all muonic 
molecular ions are three-body systems with unit charges. The energy spectra in such systems have many significant differences from known atomic spectra. In particular, any Coulomb three-body system with unit charges has a finite number of bound states [9]. The only exception to this rule is the ${ }^{\infty} \mathrm{H}_{2}^{+}$ion which has infinite number of bound states [9].

\section{OPTIMIZATION OF THE NON-LINEAR PARAMETERS IN TRIAL WAVE FUNCTIONS}

Optimization of the non-linear parameters in trial wave functions is a central part of construction of any highly accurate solution of the Schrödinger equation. By optimizing the non-linear parameters in some rapidly convergent variational expansions one can produce extremely accurate and compact wave functions for arbitrary three-body systems. Such wave functions can be used in further calculations of various bound state properties and different corrections to the non-relativistic energies of three-body systems. In this study we construct our highly accurate variational wave functions, Eq.(3), with the use of a two-stage

optimization strategy of the non-linear parameters [6]. The two-stage procedure consists of: (a) construction of a short-term booster wave function with carefully optimized non-linear parameters, and (b) quasi-random choice of the remaining non-linear parameters from a few (usually, three - five) optimal boxes (or parallelotops). To simplify the description of our optimization procedure below we shall assume that all exponents in Eq.(33) are real. In other words, all parameters $\delta_{i}, e_{i}$ and $f_{i}$ in Eq.(3) equal zero identically for $i=1, \ldots, N$. In fact, these later parameters are really needed only for highly accurate calculations of the pure adiabatic three-body systems and systems close to them. Among all muonic molecular ions $p p \mu, p d \mu, p t \mu, d d \mu, d t \mu$ and $t t \mu$ even the two heaviest systems $d t \mu$ and $t t \mu$ are not close to adiabatic systems, examples of which are the one-electron $\mathrm{DT}^{+}$and $\mathrm{T}_{2}^{+}$ions.

For muonic molecular ions considered in this study the short-term booster wave function, Eq.(3), usually includes $N_{0}=400$ - 600 basis functions (exponents) with $3 N_{0}=1200-1800$ non-linear parameters in them. All these parameters must carefully be optimized. After such an optimization the short-term booster function provides 11 - 15 exact decimal digits for each bound state energy in the considered muonic molecular ions. It appears that the overall accuracy of such short-term wave functions is much better for the protium muonic molecular ions $p p \mu, p d \mu, p t \mu$ than for the heavier ions $t t \mu$ and $d t \mu$. At the second stage 
of our optimization procedure the remaining $3\left(N-N_{0}\right)$ non-linear parameters in the wave function Eq.(3) are chosen quasi-randomly from three different boxes or parallelotops. The total energies and other bound state properties obtained with such trial wave functions depend upon the boundaries of these boxes. In reality the boundaries of these three boxes can be described [6] with the use of 28 non-linear parameters only. The numerical values of these 28 parameters were optimized approximately with the use of $N=800,1000,1200$ and 1400 basis functions [6]. These values allowed us to determine the approximate limit for each of these 28 parameters as $N \rightarrow \infty$ by extrapolation. These limiting values have been used in our final computations.

The two-stage strategy proposed in [2] and described above allows one to obtain very accurate variational wave functions based on the use of exponents in relative and/or perimetric coordinates. In particular, the overall accuracy of our results obtained in this study for all considered muonic molecular ions (see Tables I, II, III and IV below) is significantly higher than the accuracy obtained for these ions in earlier calculations. Moreover, by using this optimization strategy we expect to be able to increase the accuracy in future calculations by a factor of $\approx 10^{3}-10^{5}$ which would be sufficient for all future anticipated theoretical needs. The described two-stage optimization procedure has been used in this study for all bound $S(L=0)$ - and $P(L=1)$-states in muonic molecular ions, including all excited states. For the bound $D(L=2)$-states in heavy muonic molecular ions $(d d \mu, d t \mu$ and $t t \mu)$ we used another approach in which the short-term booster function is not constructed. However, it is clear that our current strategy for optimization of the non-linear parameters in the wave functions of the bound $D(L=2)$-states is not optimal. In future studies we want to improve this strategy and produce highly accurate results for all bound $D(L=2)$-states in muonic molecular ions.

\section{VARIATIONAL ENERGIES OF MUONIC MOLECULAR IONS}

The results of our calculations of different bound states can be found in Tables I - VI. All computations are performed in muon-atomic units, where $m_{\mu}=1, \hbar=1$ and $e=1$ and we used the following values of nuclear masses [10], [11]:

$$
\begin{gathered}
m_{\mu}=206.768262 m_{e} \quad, \quad m_{p}=1836.152701 m_{e} \\
m_{d}=3670.483014 m_{e} \quad, \quad m_{t}=5496.92158 m_{e}
\end{gathered}
$$


where $m_{e}$ designates the electron mass. Note that our highly accurate computations in this study are performed with the use of 84 - 104 decimal digits per computer word [12], [13], allowing total energies to be determined to an accuracy $\approx 1 \cdot 10^{-20}-1 \cdot 10^{-23}$ m.a.u. A natural and effective way to perform such calculations is to assume that all particle masses and corresponding conversion factors (e.g., the factor $R y$ below) are exact. Such assumptions are always made in papers on highly accurate computations in few-body systems (see, e.g., [14] and [15]). The known experimental uncertainties in particle masses and conversion factors are taken into account at the last step of calculations, when the most accurate computations are simply repeated for a few times with the use of different particle masses and conversion factors. Analogously, the lowest order relativistic and QED corrections can be determined as the expectation values of some operators computed with our non-relativistic wave functions. To avoid a substantial loss of numerical accuracy during such computations these non-relativistic wave functions must be extremely accurate.

Table I contains the total variational energies obtained for the ground $S(L=0)$-states of the non-symmetric muonic molecular ions $p d \mu, p t \mu$ and $d t \mu$. Table II includes the total energies for the excited $S^{*}(L=0)$-states of the $d d \mu, t t \mu$ and $d t \mu$ muonic molecular ions. The total energies of the ground $S(L=0)$ - and 'rotationally' excited $P(L=1)$-states of the symmetric muonic molecular ions $p p \mu, d d \mu$ and $t t \mu$ are presented in Table III - IV. Table V contains the best variational energies obtained in our computations of the $D(L=2)$-states of the $d d \mu, t t \mu$ and $d t \mu$ muonic molecular ions. Highly accurate computations of these bound $D(L=2)$-states have been performed with the use of extended arithmetic (for discussion and references, see, [15]). In earlier works the bound $D(L=2)$-states have been determined with the use of quadruple precision only. However, our current total energies determined for the bound $D(L=2)$-states are not as accurate as the total energies obtained for the bound $S(L=0)$ - and $P(L=1)$-states (see comment above).

Note also that the total energies of the $P(L=1)$-states of the non-symmetric muonic molecular ions $p d \mu, p t \mu$ and $d t \mu$ and excited $P^{*}(L=1)$-states of the $d d \mu, t t \mu$ and $d t \mu$ ions have recently been determined in [16]. The most accurate variational energies for all known 22 bound states in the set of six muonic molecular ions studied here (expressed in muonatomic units) can be found in Table VI. Note that the $F(L=3)$-state of the $t t \mu$ ion has not been re-calculated in this study, but instead it has been taken from our earlier work [2], where this state was computed with the use of quadruple precision. 
As follows from Tables I - VI the total energies obtained in this study for different bound states in six muonic molecular ions are significantly more accurate than the corresponding energies computed in earlier studies (see, e.g., [2], [14] and [15]). The current wave functions are more compact and have better overall quality than wave functions obtained in [2], [14] and [15]. They can be used for highly accurate computations of other bound state properties, including properties which contain singular expectation values.

Our variational wave functions can be used to compute some bound state properties of muonic molecular ions. A large number of bound state properties have been computed in our earlier studies (see, e.g., [6] and references therein). However, some of the bound state properties could not be determined to high numerical accuracy, due to relatively low accuracy of the wave functions used in earlier studies. It was clear that the expectation values of some nuclear-nuclear properties, e.g., all properties which include the nuclearnuclear delta-function, needed to be re-calculated with more accurate wave functions. By using highly accurate wave functions obtained in this work we have performed numerical re-calculation of a number of bound state properties for different muonic molecular ions.

The computed expectation values can be found in Tables VII and VIII. Results presented in Table VII illustrate convergence of some expectation values upon the total number $N$ of basis functions used. By comparing the expectation values computed with the use of different number(s) of basis functions we have determined the corresponding asymptotic values for $N \rightarrow \infty$. Formally, this procedure allows one to determine the number of stable decimal digits for each of the computed expectation values. Table VIII contains some bound state properties determined for the ground $S(L=0)$ - states in the $p d \mu$ and $d t \mu$ muonic molecular ions and for the excited $S(L=0)$-state in the $d t \mu$ ion.

\section{MASS DEPENDENCE OF THE TOTAL ENERGIES FOR MUONIC MOLEC- ULAR IONS}

Let us consider the problem of mass dependence for the total energies of muonic molecular ions. Briefly, this problem is formulated as follows. The Hamiltonian $H$ of any muonic molecular ion, Eq.(1), contains three different masses $m_{a}, m_{b}, m_{\mu}$. These particle masses are the subject of constant experimental revision. Furthermore, various authors often use slightly different particle masses in their calculations. For highly accurate computations this 
means the almost constant necessity of re-calculation of the energies and corresponding wave functions. In fact, all masses of particles which form muonic molecular ions are currently known to relatively high experimental accuracy, i.e. all possible 'mass-corrections' must be very small. This means that such corrections can be considered by using various methods of perturbation theory. Nevertheless, it is very interesting in some cases to evaluate corrections produced by the corresponding 'mass shift'. The most important bound state property is the total energy $E$. The first order variation of $E$ with the particle masses is written in the form

$$
E_{\text {new }}=E_{\text {our }}+\alpha\left[\frac{m_{\mu}(\text { new })}{m_{t}(\text { new })}-\frac{m_{\mu}(\text { our })}{m_{t}(\text { our })}\right]+\beta\left[\frac{m_{\mu}(\text { new })}{m_{d}(\text { new })}-\frac{m_{\mu}(\text { our })}{m_{d}(\text { our })}\right]
$$

in the case of the $d t \mu$ ion. In Eq.(6) and Eq.(77) below all energies must be expressed in the same units, e.g., in atomic units or in muon-atomic units reduced to the same muon mass. The same formula can be written for any non-symmetric muonic molecular ion. In such cases we always have $\alpha \geq \beta$, if the coefficient $\alpha$ corresponds to the mass shift produced by the heaviest nucleus. For symmetric muonic molecular ions, e.g., for the $d d \mu$ ion, the analogous formula is

$$
E_{\text {new }}=E_{\text {our }}+\alpha\left[\frac{m_{\mu}(\text { new })}{m_{d}(\text { new })}-\frac{m_{\mu}(\text { our })}{m_{d}(\text { our })}\right]
$$

In these equations the notation 'our' denotes the mass value used in this study, while the notation 'new' designates a different mass value, e.g. from some work performed in the future. In general, the numerical values of the coefficients $\alpha$ and $\beta$ in Eq.(6) (also called the mass gradients) are determined from separate energy calculations with different masses. In our earlier works we have used a very simple approach based on four additional calculations with different masses for non-symmetric muonic molecular ions [17]. For symmetric muonic molecular ions one has to perform at least two additional calculations with the two different mass ratios. This method is simple, but it is not very accurate.

Recently, we have developed a more accurate procedure. To describe this procedure let us consider the ground $P(L=1)$-state in the $d d \mu$ ion. The mass ratio $\frac{m_{\mu}(\text { our })}{m_{d}(\text { our })}$ is designated below as $x_{0}$, while the notation $h$ stands for the difference $\frac{m_{\mu}(\text { new })}{m_{d}(\text { new })}-\frac{m_{\mu}(\text { our })}{m_{d}(\text { our })}$ used in Eq.(7). In these notations the total energies $E_{\text {our }}$ and $E_{\text {new }}$ are $E_{\text {our }}=E\left(x_{0}\right)$ and $E_{\text {new }}=E\left(x_{0}+h\right)$, respectively. The absolute value of $h$ is assumed to be small. Our new method for calculation of the mass gradient $\alpha$ is based on calculation of the four ground state energies $E\left(x_{0}-2 h\right), E\left(x_{0}-h\right), E\left(x_{0}+h\right)=E_{\text {new }}$ and $E\left(x_{0}+2 h\right)$. These four energies 
are determined in calculations performed with the use of the maximal number of basis functions (at the maximal dimension). No re-optimization of non-linear parameters in the wave function is required during such additional computations. The 'mass gradient' $\alpha$ in Eq.(7) can now be computed with the use of the formula

$$
\alpha=\frac{1}{12 h}\left[E\left(x_{0}-2 h\right)-8 E\left(x_{0}-h\right)+8 E\left(x_{0}+h\right)-E\left(x_{0}+2 h\right)\right]+\frac{h^{4}}{30} A
$$

where $A$ is a numerical parameter of order of unity. It can be shown that this parameter

equals the fifth order derivative of the total energy $E$ with respect to the mass ratio $\frac{m_{\mu}}{m_{d}}$ computed in some point between $x_{0}-2 h$ and $x_{0}+2 h$. The formula, Eq.(8), has a very good numerical accuracy in actual applications to all bound states, except weakly-bound states (discussed below).

The results of numerical calculations of the mass gradients $\alpha$ in Eq. (88) for the $P(L=$ 1)-states in the symmetric $p p \mu, d d \mu$ and $t t \mu$ muonic molecular ions are presented in Table IX. Table IX also contains the results for the excited $P^{*}(L=1)$-state in the $t t \mu$ ion. All these energies have been re-calculated to the 'standard' muon mass $m_{\mu}=206.768262 m_{e}$. This Table also contains all intermediate energies $E\left(x_{0}-2 h\right), E\left(x_{0}-h\right), E\left(x_{0}+h\right)$ and $E\left(x_{0}+h\right)$ needed in such calculations. As follows from Eq.(8) our mass gradients $\alpha$ are accurate to 11 - 13 decimal digits. The same procedure can be used for other bound states in muonic molecular ions.

\section{A. Mass dependence for the weakly-bound states}

The mass dependence of the total and binding energies for weakly bound states cannot be investigated with the use of the method described above, since the total energies $E\left(x_{0}+\right.$ $h)$ and $E\left(x_{0}+2 h\right)$ needed in Eq.(8) can correspond to unbound states. This indicates a necessity to study the mass dependence of the total and binding energies for weakly bound states. Formally, to answer this question one needs to perform a number of calculations with different particle masses. For simplicity, let us discuss the weakly bound excited $P^{*}(L=$ 1) -state in the $d t \mu$ ion. If the muon mass $m_{\mu}$ and the muon-deuterium mass ratio $\tau=\frac{m_{\mu}}{m_{d}}$ increase, then the $P^{*}(L=1)$-state in the $d t \mu$ ion will be less and less bound. Finally, at some critical values of $m_{\mu}$ and $\tau$ the $P^{*}(L=1)$-state in the $d t \mu$ ion will become unbound.

The approach based on the use of 'conventional wisdom' allows one quickly to find the 
numerical value of the critical muonic mass. Indeed, the Hamiltonian of muonic molecular ions, Eq.(21), is a linear function of the muonic mass $m_{\mu}$ and/or the mass ratio $\tau=\frac{m_{\mu}}{m_{d}}$. Therefore, by performing calculations of the total energy of some bound state for different muonic masses $m_{\mu}$ one finds the dependence $E\left(m_{\mu}\right)$, or $E\left(m_{\tau}\right)$. The next step is to determine the critical mass for which $E\left(m_{\mu}\right)=-\frac{1}{2} \frac{m_{\mu} m_{t}}{m_{\mu}+m_{t}}$, i.e. the binding energy of this state equals zero. For a very short interval of variation of $m_{\mu}$ the function $E\left(m_{\mu}\right)$ is almost a linear function upon the muonic mass $m_{\mu}$, then it is relatively easy to obtain an approximate value of the critical muonic mass.

This approach was used in some earlier works, but it produces incorrect results in applications to the bound states which can disappear (as bound states) during variations of some physical parameter, e.g., the mass of the particle. For such states one needs to use an alternative approach described in [18]. In this method the explicit expression for the total energy of the two-body 'almost unbound' system takes the form

$$
\varepsilon=-\frac{\pi^{2}}{16} \frac{\left(|U|-\left|U_{1}\right|\right)^{2}}{\left|U_{1}\right|}=-\frac{\pi^{2} \hbar^{2}}{32} V^{2} \frac{\left(m_{1}-m\right)^{2}}{m^{2} m_{1}^{2}}
$$

where $U=\frac{\hbar^{2}}{2 m} V$ is the effective two-body potential in the two-body $d \mu+d$ system. The value $U_{1}$ corresponds to the mass $m_{1}$ at which $\varepsilon=0$. The difference of masses $m_{1}$ and $m$ is assumed to be small in comparison with each of the two masses $\left(m_{1}\right.$ and $\left.m\right)$. The formula, Eq.(91), predicts a quadratic dependence of the energy $\varepsilon$ upon the mass difference $\Delta=m_{1}-m$, i.e. $\varepsilon\left(m_{1}, m\right)=\varepsilon\left(m_{1}, \Delta\right) \approx a \cdot \frac{\Delta^{2}}{m_{1}^{4}}$. Although the actual $\varepsilon\left(m_{1}, \Delta\right)$ dependence must be described by the formula Eq.(9) to good accuracy, in reality however it is difficult to obtain the quadratic dependence for the $\varepsilon\left(m_{1}, \Delta\right.$ ) function (on the parameter $\Delta$ ) by using the results of numerical calculations with the same number of basis functions. It is clear that in accurate calculations of bound states with $\Delta \rightarrow 0$ one needs to increase the total number of basis functions and constantly re-optimize the non-linear parameters of the method. If such conditions are obeyed, then we can observe the expected quadratic dependence of the binding energy $\varepsilon$ on $\Delta$. Otherwise, from the results of such calculations one can see only an approximate linear dependence.

Note that for real muonic molecular ions the total energy of the two-body system $\varepsilon$ given in Eq.(99) is, in fact, the binding energy of the three-body ion, e.g., the $d t \mu$ ion, which corresponds to the lowest-by-energy decay channel: $(d t \mu)^{+}=t \mu$ (ground state) $+d^{+}$. For the $d t \mu$ ion the mass $m$ in Eq.(9) is the 'reduced' mass $m=\frac{\left(m_{t}+m_{\mu}\right) m_{d}}{m_{t}+m_{\mu}+m_{d}}$, while $m_{1}=\frac{\left(m_{t}+\tilde{m}_{\mu}\right) m_{d}}{m_{t}+\tilde{m}_{\mu}+m_{d}}$ 
is the 'threshold' mass, i.e. the reduced muonic mass for which the binding energy equals zero.

The results of our calculations for the $P^{*}(L=1)$-state in the $d d \mu$ ion can be found in Table X. In these calculations we have increased (at each step) the mass of the $\mu^{-}$muon (see above) by one electron mass $m_{e}$. As follows from Table $\mathrm{X}$ the binding energy of the $P^{*}(L=1)$-state in the $d d \mu$ ion decreases to zero. The value of muon mass at which the corresponding bound state becomes unbounded is called the threshold mass. For the $d d \mu$ ion such a muon threshold mass is designated as $\tilde{m}_{\mu}$. In calculations for Table $\mathrm{X}$ we have used $N=3300$ basis functions. The $\varepsilon\left(m_{1}, \Delta\right)$ dependence which follows from the results of these calculations can be represented as a linear function. The threshold value of $\tilde{m}_{\mu}$ found from this linear dependence approximately equals to the value $\tilde{m}_{\mu}=m_{\mu}+7.95 m_{e} \approx$ $214.718262 m_{e}$. As follows from Table $\mathrm{X}$ the binding energy of the $P^{*}(L=1)$-state in the $d d \tilde{\mu}$ ion is $\approx-0.00002551843452 \mathrm{eV}$. This energy was obtained with $N=3300$ basis functions. However, if we take 3840 basis functions, then the binding energy of the $P^{*}(L=1)$-state in the same $d d \tilde{\mu}$ ion is $\approx-0.00007865184962 \mathrm{eV}$. i.e., it corresponds to the state which is slightly better bound. The muon threshold mass is always shifted to larger values of $\tilde{m}_{\mu}$ if the total number of basis functions increases. This example explains the occurence of what is often called "running" mass threshold, a non-physical effect known to exist in numerical calculations of any weakly-bound state. It is interesting to note that the muon mass $m_{\mu}=214.718262 m_{e}$ exceeds our 'standard' muon mass $\left(206.768262 m_{e}\right)$ already by $\approx$ $4 \%$, but the $P^{*}(L=1)$-state in the $d d \mu$ ion is still bound (earlier estimations for such a mass deviation were around $0.5-1 \%$ ).

Analogous results for the weakly bound $P^{*}(L=1)$-state in the $d t \mu$ ion can be found in Table XI. In computations performed for this Table we varied only the muon mass $m_{\mu}$, while the deuterium and tritium masses have not been changed. At such conditions the muon threshold mass was found to be equal $\tilde{m}_{\mu} \approx m_{\mu}+1.99 m_{e}$. It is clear that the binding energy of the $P^{*}(L=1)$-state in the $d t \mu$ ion is the function of the two independent mass ratios $\frac{m_{\mu}}{m_{d}}$ and $\frac{m_{\mu}}{m_{t}}$. Therefore, Table XI gives only an approximate picture of how the total and binding energies (in eV) of the $P^{*}(L=1)$-state in the $d t \mu$ ion vary when the muon mass changes. To study the pre-threshold mass dependence of the weakly bound $P^{*}(L=1)$-state in the $d t \mu$ ion in detail one also needs to consider changes of the deuterium and tritium masses. Such calculations, however, are very difficult to perform, since they require substantial computer 
resourses.

\section{MUON STICKING PROBABILITIES}

Originally, all numerical computations of bound states in muonic molecular ions were motivated by various problems of muon-catalyzed nuclear fusion. In fact, the bound state computation of muonic molecular ions is only one of many problems which must be solved before we can discuss a possibility to use muon-catalyzed nuclear fusion for energy production and for other purposes. It is clear that the most interesting and promising case is the muon catalysis of the $(d, t)$-nuclear reaction in the $d t \mu$ ion. A central problem here is to determine the muon sticking probability during the nuclear reaction $d t \mu={ }^{4} \mathrm{He}+\mu+n$ since the numerical value of this coefficient essentially determines the feasibility of using muon catalysis of nuclear fusion reactions for energy production purposes. Let us evaluate the muon sticking probabilities for this ion by assuming that the nuclear $d t$-fusion occurs only in the two $S(L=0)$-states (ground and excited), ignoring the possibility of nuclear fusion in the $P(L=1), P^{*}(L=1)$ and $D(L=2)$ bound states of the $d t \mu$ ion.

The analytical expression for the muon sticking probability for the bound $S(L=0)$-state (initial state is designated with the subscript in; final state by $f i$ ) takes the form [6] (see also [19] and [20])

$$
P_{i n ; f i}=4 \pi(2 \ell+1)\left|\int_{0}^{+\infty} \phi_{i n}\left(a^{-1} r\right) j_{\ell}(Q r) R_{n \ell}(r) r^{2} d r\right|^{2}
$$

where $n$ and $\ell$ are the appropriate principal and angular quantum numbers for the final hydrogen-like $\left({ }^{4} \mathrm{He} \mu\right)^{+}$ion with radial function $R_{n \ell}(r)$. The choice of the factor $a$ in Eq.(10) is discussed below. The $j_{\ell}(Q r)$ function is the spherical Bessel function (see e.g., [21]):

$$
j_{\ell}(x)=\sqrt{\frac{\pi}{2 x}} J_{\ell+\frac{1}{2}}(x)
$$

The factor $Q$ is

$$
Q=m_{\mu} v=\sqrt{\frac{2 m_{n} \Delta E}{\left(1+M_{4}\right)\left(1+m_{n}+M_{4}\right)}}=5.825011748
$$

where $m_{n}=1838.683662 m_{e}$ is the neutron's mass, $\Delta E$ is the total energy release during the nuclear $(d, t)$-reaction and $M_{4}=7294.2296 m_{e}$ is the mass of the ${ }^{4}$ He nucleus.

In the formulas presented above $\phi_{i n}\left(a^{-1} r\right)$ is the initial 'post-process' wave function, i.e. the wave function of the system which arises when the sudden process (i.e. nuclear fusion) 
is over. The function $\phi_{i n}\left(a^{-1} r\right)$ can be found from the bound state wave function $\Psi$ of the initial three-body system. For instance, in the case of nuclear fusion in the $S(L=0)$-state of the $d t \mu$ muonic molecular ion one finds:

$$
\phi_{i n}\left(r_{32}\right)=\delta_{21} \cdot \Psi\left(r_{32}, r_{31}, r_{21}\right)=\Psi\left(r_{32}, r_{32}, 0\right)=\sum_{i=1}^{N} C_{i} \exp \left(-\left(\alpha_{i}+\beta_{i}\right) r_{32}\right),
$$

where $\delta_{21}$ is the nuclear delta-function and $C_{i}$ are the linear variational parameters from Eq.(1). These coefficients have been determined during numerical solution of the Schrödinger equation for the initial three-body system (see Section IV). Note also, that after the 'sudden' nuclear fusion the new ${ }^{4} \mathrm{He}$ nucleus arises at the same point '2'. This does not change the relative $r_{32}$ coordinate which is mass independent. After the nuclear reaction the $r_{32}$ coordinate becomes the helium-muonic relative coordinate. In two-body atomic problems, however, it is more convenient to use the mass-weighted coordinate $r$. The relation between the relative $r_{32}$ coordinate and mass-weighted $r$ coordinate (which corresponds to the heliummuonic ion) is written in the form:

$$
r=a r_{32}=\frac{m_{\mu} M_{4}}{m_{\mu}+M_{4}} r_{32} \quad \text { or } \quad r=\frac{M_{4}}{1+M_{4}} r_{32}
$$

where in muon-atomic units $m_{\mu}=1$ and $M_{4}$ is the nuclear mass (in muon-atomic units) of the ${ }^{4} \mathrm{He}$ nucleus. Finally, the initial wave function $\phi_{i n}\left(a^{-1} r\right)$ takes the form:

$$
\phi_{i n}\left(a^{-1} r\right)=\sum_{i=1}^{N} C_{i} \exp \left(-\left(\alpha_{i}+\beta_{i}\right) a^{-1} r\right)
$$

where $a^{-1}=\frac{1+M_{4}}{M_{4}}$. For the muon and nuclear masses indicated above, one finds that $a^{-1}\left({ }^{4} H e\right)=1.028346555$.

The formulas given above allow us to determine the muon sticking probabilities for the ground and excited $S(L=0)$-states in the $d t \mu$ ion. In these calculations we have used our best variational wave functions, as obtained in this study. After a number of calculations we have evaluated the total muon sticking probabilities $P_{s}$ for the ground and excited $S(L=0)$ states in the $d t \mu$ ion. These two numerical values are close to each other. In fact, we have found that each of these two values $\left(P_{s}\right)$ is bounded in the interval $0.008923(3) \leq P_{s} \leq$ 0.008938 (3) (for both bound $S$-states). This means that the total number of $d t$-nuclear reactions catalyzed by one $\mu^{-}$muon is $\kappa=P_{s}^{-1} \approx 112$. This means that before its decay one $\mu^{-}$muon can catalyze up to 112 nuclear reactions of $d t$-fusion. This number is used in the next Section to discuss the possibility of exploiting muon catalyzed fusion for energy production purposes. 


\section{CURRENT STATUS OF MUON-CATALYZED NUCLEAR FUSION}

Our evaluation of the factor $\kappa=P_{s}^{-1} \approx 112$ performed above is based on an assumption that all nuclear $(d, t)$-fusion reactions proceed from the bound $S$-states of the $d t \mu$ muonic molecular ion. This factor does not take into account the stripping of muons from rapidly moving He nuclei and a possibility of nuclear $(d, t)$-fusion in the bound $P$ - and $D$-states of the $d t \mu$. Formally, an average time required to complete one $(d, t)$-fusion reaction in any of the $P$ - and/or $D$-states is substantially longer than the analogous time for the $S-$ state(s). In fact, such a 'reaction time' is comparable with the transition time which is needed to complete all transitions from the bound $P(L=1), P^{*}(L=1)$ and $D(L=2)$ states into the corresponding $S(L=0)$-state(s). This directly follows from the fact that actual deuterium-tritium distances are larger in the $P$ - or $D$-states of the $d t \mu$ ion than in the $S$-states. Briefly, this means that all $d t \mu$ ions originally formed in the $P$ - and $D$-states will emit radiation and make transitions into the corresponding $S(L=0)$-states. In other words, a possibility of nuclear fusion from the 'rotationally excited' bound $P-$ and $D-$ states can be ignored and all reactions of the nuclear $(d, t)$-fusion in the $d t \mu$ ion can reasonably to assume to proceed only from the two bound $S$-states of the $d t \mu$ ion. Let $P$ be exact sticking probability of the muon in the $d t \mu$ ion. The analogous value $P_{s}$ is the muon sticking probability determined for the same $d t \mu$ ion, but only for its bound $S(L=0)$-states. As follows from the discussion above we can replace the factor $\kappa=P^{-1}$ by the approximate value $\kappa \approx P_{s}^{-1}$.

There are also a few other corrections which can change the numerical value of the factor $\kappa=P_{s}^{-1} \approx 112$. The largest of such corrections corresponds to the 'muon stripping' during collisions of the fast $\left({ }^{4} \mathrm{He} \mu\right)^{+}$ion with neutral hydrogen molecules. However, even such a correction cannot change the predicted value of $\kappa$ by $40 \%$ [1]. In other words, the maximal value of $\kappa$ is $\approx 160$ - 170 . On the other hand, to reach break-even, i.e. to compensate for the energy spent for creation of one $\mu$ muon $(\approx 8000 \mathrm{MeV}$ [1], [22] ), one muon needs to catalyze at least 2285 nuclear $d t$-reactions. In this evaluation we have ignored all possible energy losses and assumed $100 \%$ efficiency for each muon. In reality any thermal-to-electrical conversion has only $\sim 30 \%$ efficiency and only $\sim 70 \%$ of all muons can produce the maximal number of fusion reactions. With all these corrections one finds that the factor $\kappa$ must be $\approx 8,000-11,000$ to reach break-even. Such values are $\approx 65$ times larger than 
the maximal value of $\kappa$ which has been measured experimentally $(\kappa \approx 150)$. If somehow in future experiments the numerical value of $\kappa$ will be increased up to 500, even then it will be $\approx 20$ times smaller the value which is needed to reach break-even. This indicates clearly that muon catalysis of nuclear reactions cannot be used for energy production purposes.

It should be mentioned that originally the idea to use $\mu^{-}$-muons for production of repetitive nuclear reactions between light nuclei of hydrogen isotopes was proposed more than sixty years ago [23]. Based on an obvious chemical analogy these processes were called the muonic catalysis of nuclear reactions. It was confirmed in [24] experimentally by observing two consecutive $(p, d)$-nuclear reactions catalyzed by the same muon. The first numerical computations of the bound states in three-body muonic molecular ions were performed by Belyaev et al in 1959 [25] who found only 20 bound states in six ions $p p \mu, p d \mu, p t \mu, d d \mu, d t \mu$ and $t t \mu$. The overall accuracy of the procedure used in [25] was very low and the authors could not confirm the boundness of the excited $P^{*}(L=1)$-states (or (1,1)-states) in the $d d \mu$ and $d t \mu$ ions. It was concluded only that, if such states are bound, then they are very weakly bound. The binding energy of these two states was expected to be smaller than 4.5 $e V$, i.e. smaller than the binding energy of a typical molecule. Immediately after publication of [25] an intense stream of speculations started about a possible interference (or resonance) between the formation of excited $P^{*}(L=1)$-states (or (1,1)-states) in the $d d \mu$ and $d t \mu$ muonic molecular ions and different atomic/molecular processes in surrounding molecules (see, e.g., [26] and references there in). Finally, in a few experimental studies performed by Bystritskii et al (see [27] and [28] and references therein) it was shown that one muon can catalyze approximately 10 - $20(d, d)$-nuclear reactions in liquid deuterium $\left(\mathrm{D}_{2}\right)$ and 90 - 110 $(d, t)$-reactions in the liquid equimolar deuterium-tritium mixture $\left(\mathrm{D}_{2}: \mathrm{T}_{2}=1: 1\right)$. Such very large numbers of nuclear reactions catalyzed by one muon can be explained only by the resonance (or very fast) formation of $d d \mu$ and $d t \mu$ muonic molecular ions. Correspondingly, these processes were called 'resonance' muon-catalyzed fusion of nuclear reactions, in contrast with the 'regular' muon-catalyzed fusion observed in [24].

In experiments performed in 1980's the total number of nuclear reactions catalyzed by one muon (i.e. the numerical value of the factor $\kappa$ defined above) for the equimolar deuteriumtritium mixture were evaluated a $\mathrm{s} \approx 150$ (see discussion and references in [1]). This value is $\approx 15$ times smaller than the value which is needed for theoretical break-even and $\approx 65$ times smaller than necessary for actual break-even. Therefore, we have to conclude that all 
discussion of the 'bright future' for applications of the resonance muon-catalized future for the energy production purposes appears to be groundless.

\section{CONCLUSION}

We have considered the problem of highly accurate calculations of bound states in the three-body muonic molecular ions $p p \mu, p d \mu, p t \mu, d d \mu, d t \mu$ and $t t \mu$. The study of bound state spectra in the muon-molecular ions is of interest for solving some theoretical problems and in a number of applications. In fact, our present knowledge of the bound state spectra in Coulomb three-body systems with unit charges is essentially based on knowledge of the spectra of the muonic molecular ions. Note that all muonic molecular ions can easily be created in real experiments and their various properties can be measured quite accurately. From a certain point of view, theoretical and experimental study of these ions is more interesting and informative than the traditional analysis of atomic three-body (i.e. twoelectron) systems.

The results of variational computations of the total energies for various bound states in the various muonic molecular ions are presented in Tables I - VI. Table VI contains the most accurately known predictions for variational energies in muon-atomic units for each of 22 known bound states for muonic molecular ions. The accuracy of these total bound state energies significantly exceeds the accuracy achieved in earlier studies. It should be mentioned that first variational calculations of muonic molecular ions started almost 45 years ago [29], [30], [31], [32]. In these works only $S(L=0)-$ and $P(L=1)$-states of muonic molecular ions were considered. It is interesting to note that at that time the nonvariational calculations (see, e.g., [33] and references therein) of muonic molecular ions had a comparable overall accuracy for many bound $S(L=0)$ - and $P(L=1)$-states in muonic molecular ions. In addition to this, the non-variational methods allow one to determine the total energies of the bound $D(L=2)$ - and $F(L=3)$-states in muonic molecular ions [33].

Our first variational calculations of muonic molecular ions started 25 years ago [34]. In particular, the first successful variational computations of the weakly bound $P^{*}(L=$ 1)-states in the $d d \mu$ and $d t \mu$ ions were performed in our work [34] and also in [35]. However, at that time we could not compute the bound $D$ - and $F$-states in the $d d \mu, d t \mu$ and $t t \mu$ ions. Furthermore, our maximal accuracy achieved at that time was relatively low. Currently, the 
same energies for all $S(L=0)$ - and $P(L=1)$-states in muonic molecular ions obtained in [34] can be reproduced with the use of only 20 - 30 exponential basis functions in Eq.(3)), with carefully chosen non-linear parameters $\alpha_{i}, \beta_{i}$ and $\gamma_{i}$ in each basis function. The first variational computations of the bound $D(L=2)$-states in the $d t \mu, d d \mu$ and $t t \mu$ ions were performed in 1986 [36], while analogous calculations of the $F(L=3)$-state in the $t t \mu$ ion

were conducted 15 years later [2]. The bound $D$-state in the $d t \mu$ ion was also calculated (variationally) by Kamimura in 1988 [37].

By using our highly accurate wave functions we have determined the expectation values of some bound state properties of muonic molecular ions. We also discuss the problem of mass shifts in muonic molecular ions, including the case of weakly bound states. A separate (but very important) problem is to evaluate the muon sticking probabilities for the $S(L=0)$-states in the $d t \mu$ ion. By using these probabilities we estimated the total number of the reactions of $(d, t)$-fusion which can be catalized by one $\mu^{-}$muon in a liquid equimolar $\mathrm{D}_{2}: \mathrm{T}_{2}$ mixture. The current status of the 'resonance' muon-catalyzed nuclear fusion is briefly discussed. It is shown that this process cannot be used for energy production purposes.

\section{Acknowledgements}

It is a pleasure to acknowledge the University of Western Ontario for financial support.

[1] L.I. Men'shikov and L.N. Somov, Sov. Phys. Uspekhi 33, 616 (1990).

[2] A.M. Frolov, J. Phys. B 34, 3813 (2001).

[3] A.M. Frolov and D.M. Bishop, Phys. Rev. A 45, 6236 (1992).

[4] I.I. Sobelman, Introduction to the Theory of Atomic Spectra, (Pergamon Press, Oxford-New York, 1972).

[5] M. Born and J.R. Oppenheimer, Ann. Phys. 84, 457 (1927).

[6] A.M. Frolov, Phys. Rev. E 64, 036704 (2001).

[7] D.A. Varshalovich, A.N. Moskalev and V.K. Khersonskii, Kvantovaya Teoriya Uglovogo Momenta, [Quantum Theory of Angular Momentum], Nauka, Moscow (1975) (in Russian).

[8] A.M. Frolov, Zh. Eksp. Teor. Fiz. 92, 1959 (1987) [Sov. Phys. JETP 92, 1100 (1987)].

[9] A.M. Frolov, Phys. Rev. A 59, 4270 (1999). 
[10] The NIST Reference on Constants, Units and Uncertainty, see: http://physics.nist.gov/cuu/Constants/index.html

[11] CRC Handbook of Chemistry and Physics, 85th Edition, Ed. D.R. Lide, (CRC Press, Inc., Boca Raton, Florida, 2004).

[12] D.H. Bailey, ACM Trans. Math. Soft. 21, 379 (1995).

[13] D.H. Bailey, Computing in Science and Engineering, 2, 24 (2000).

[14] S. Kilic, J.-P. Karr and L. Hilico, Phys. Rev. A 66, 22101 (2002).

[15] D.H. Bailey and A.M. Frolov, J. Phys. B 35, 4287 (2002).

[16] A.M. Frolov and D.M. Wardlaw, Phys. Rev. A 81, 062504 (2010).

[17] A.M. Frolov, H.H. Smith, Jr. and D.M. Bishop, Phys. Rev. A 49, 1686 (1994); ibid, 51, 3636 (1995).

[18] A.I. Baz, Ya.B. Zel'dovich and A.M. Perelomov, Scattering, Reactions and Decays in Quantum Mechanics, (Israel Program for Scientific Translations, Jerusalem (1969)).

[19] C.-Y. Hu, Phys. Rev. A 34, 2536 (1986).

[20] C.-Y. Hu, G.M. Hale and J.S. Cohen, Phys. Rev. A 49, 4481 (1994).

[21] L.D. Landau and E.M. Lifshitz, Quantum Mechanics. Non-relativistic Theory, (3rd. ed., Oxford, England, Pergamon Press (1977)), Chp. XIV.

[22] Yu.N. Petrov, Nature 285, 466 (1980).

[23] F.C. Frank, Nature 160, 525 (1947).

[24] L.W. Alvarez, H. Bradner, F.S. Crawford Jr., J.A. Crawford, P. Falk-Vairant, M.L. Good et al, Phys. Rev. 105, 1127 (1957).

[25] V.B. Bel'jaev, S.S. Gerstein, B.N. Zakhariev and S.P. Lomnev, Zh. Eksp. Teor. Fiz. 37, 1652 (1959) [Sov. Phys. JETP 10, 1171 (1959)].

[26] Ya.B. Zel'dovich and S.S. Gerstein, Uspekhi Fiziki 71, 581 (1960).

[27] V.M. Bysrtitskii, V.P. Dzhelepov, Z.V. Ershova, V.G. Zinov, V.K. Kapyshev et al, Pis'ma Zh. Eksp. Teor. Fiz. 31, 249 (1980) [Sov. Phys. JETP Letters 31, 228 (1980)].

[28] V.M. Bysrtitskii, V.P. Dzhelepov, Z.V. Ershova, V.G. Zinov, V.P. Kapyshev et al, Zh. Eksp. Teor. Fiz. 80, 1700 (1981) [Sov. Phys. JETP 53, 877 (1981)].

[29] A. Halpern, Phys. Rev. Lett., 13, 660 (1964).

[30] B.P. Carter, Phys. Rev. 141, 863 (1966).

[31] B.P. Carter, Phys. Rev. 165, 139 (1968). 
[32] L.M. Delves and T. Kalotas, Australian J. Phys. 21, 1 (1968).

[33] S.I.Vinitskii, V.S. Melezhik, L.I. Ponomarev, I.V. Puzynin, T.P. Puzynina, L.N. Somov and N.F. Truskova, Zh. Eksp. Teor. Fiz. 79, 698 (1980) [Sov. Phys. JETP 52, 353 (1980)].

[34] A.M. Frolov and V.D. Efros, Pizma v Zh. Eksp. Teor. Fiz., 39, 449 (1984) [Sov. Phys. JETP Lett. 39, 544 (1984)].

[35] A.K. Bhatia and R.J. Drachman, Phys. Rev. A 30, 2138 (1984).

[36] A.M. Frolov, Yad. Fiz. 44, 1367 (1986) [Sov. J. Nucl. Phys. 44, 888 (1986)].

[37] M. Kamimura, Phys. Rev. A 38, 621 (1988). 
TABLE I: The total energies $(E)$ of the ground bound $S(L=0)$-states in the non-symmetric muonic molecular ions in muon-atomic units $\left(m_{\mu}=1, \hbar=1, e=1\right) . N$ designates the number of basis functions used in Eq.(2).

\begin{tabular}{lllll}
\hline \hline$N$ & $E(p d \mu)$ & $E(p t \mu)$ & $E(d t \mu)$ \\
\hline 3300 & -0.512711796501514087 & -0.519880089782919741 & -0.538594975061413075 \\
3500 & -0.512711796501514175 & -0.519880089782919852 & -0.538594975061413084 \\
3700 & -0.512711796501514250 & -0.519880089782919605 & -0.538594975061413089 \\
3840 & -0.512711796501514283 & -0.519880089782920006 & -0.538594975061413095 \\
\hline$A$ & $-0.51271179650151455(1)$ & $-0.5198800897829210(5)$ & $-0.5385949750614133(2)$ \\
\hline$E^{a}$ & $-0.512711796501509[2]$ & $-0.519880089782914[2]$ & $-0.538594975061413[2]$ \\
$E^{a}$ & $-0.5127117965008[17]$ & $-0.5198800897819[17]$ & $-0.53859475058[17]$ \\
\hline \hline
\end{tabular}

${ }^{(a)}$ The best variational energies known from earlier calculations.

TABLE II: The total energies $(E)$ of the excited bound $S(L=0)$-states in the muonic molecular ions in muon-atomic units $\left(m_{\mu}=1, \hbar=1, e=1\right) . \quad N$ designates the number of basis functions used in Eq.(2).

\begin{tabular}{|c|c|c|c|}
\hline$N$ & $E(d d \mu)$ & $E(d t \mu)$ & $E(t t \mu)$ \\
\hline 3300 & -0.479706380368904996317 & -0.488065357851706559 & -0.496762894249561323055 \\
\hline 3500 & -0.479706380368904996742 & -0.488065357851706622 & -0.496762894249561323901 \\
\hline 3700 & -0.479706380368904997024 & -0.488065357851706665 & -0.496762894249561324665 \\
\hline 3840 & -0.479706380368904997240 & -0.488065357851706683 & -0.496762894249561325040 \\
\hline$A$ & $-0.4797063803689049978(2)$ & $-0.48806535785170680(5)$ & $-0.496762894249561327(1)$ \\
\hline$E^{a}$ & $-0.47970638036890425[2]$ & $-0.488065357851705[2]$ & $-0.49676289424956131[2]$ \\
\hline$E^{a}$ & -0.479706380368 [17] & $-0.488065357841[17]$ & -0.496762894248 [17] \\
\hline
\end{tabular}

${ }^{(a)}$ The best variational energies known from earlier calculations. 
TABLE III: The total energies $(E)$ of the bound $S(L=0)$-states in the symmetric muonic molecular ions in muon-atomic units $\left(m_{\mu}=1, \hbar=1, e=1\right) . N$ designates the number of basis functions used in Eq.(2).

\begin{tabular}{lllll}
\hline \hline$N$ & $E(p p \mu)$ & $E(d \mu)$ & $E(t \mu)$ \\
\hline 3300 & -0.49438682024893480876255 & -0.531111 & 13540238644865 & -0.546374225613816728844 \\
3500 & -0.49438682024893480876280 & -0.53111113540238644961 & -0.546374225613816728849 \\
3700 & -0.49438682024893480876289 & -0.53111113540238645059 & -0.546374225613816728855 \\
3840 & -0.49438682024893480876294 & -0.53111113540238645122 & -0.546374225613816728856 \\
\hline$A$ & $-0.49438682024893480876325(10)$ & $-0.531111135402386455(2)$ & $-0.54637422561381672890(3)$ \\
\hline$E^{a}$ & $-0.49438682024893454694[2]$ & $-0.5311111354023863745[2]$ & $-0.54637422561381671[2]$ \\
$E^{a}$ & $-0.49438682024858[17]$ & $-0.531111135402[17]$ & $-0.546374225598[17]$ \\
\hline \hline
\end{tabular}

${ }^{(a)}$ The best variational energies known from earlier calculations.

TABLE IV: The total energies $(E)$ of the bound $P(L=1)$-states in the symmetric muonic molecular ions in muon-atomic units $\left(m_{\mu}=1, \hbar=1, e=1\right) . N$ designates the number of basis functions used in Eq.(2).

\begin{tabular}{|c|c|c|c|}
\hline$N$ & $E(p p \mu)$ & $E(d d \mu)$ & $E(t t \mu)$ \\
\hline 3300 & -0.468458 436 303 38526811 & -0.513623956792681889 & -0.533263449820383210 \\
\hline 3500 & -0.468 45843630338527262 & -0.513623956792681896 & -0.533263449820383224 \\
\hline 3700 & -0.468 45843630338527622 & -0.513 623956792681901 & -0.533263449820383236 \\
\hline 3840 & -0.46845843630338527858 & -0.513623956792681905 & -0.533263449820383242 \\
\hline$A$ & $-0.46845843630338535(5)$ & $-0.51362395679268195(2)$ & $-0.53326344982038328(2)$ \\
\hline$E^{a}$ & -0.4685843630338344 [2] & $-0.51362395679268025[2]$ & $-0.5332634498203766[2]$ \\
\hline$E^{a}$ & -0.468584363033 [17] & -0.5136239567926 [17] & -0.5332634498176 [17] \\
\hline
\end{tabular}

${ }^{(a)}$ The best variational energies known from earlier calculations. 
TABLE V: The total energies $(E)$ of the bound $D(L=2)$-states of the the $d d \mu, t t \mu$ and $d t \mu$ muonic molecular ions in muon atomic units. $N$ designates the number of basis functions used in Eq.(2).

\begin{tabular}{|c|c|c|c|c|}
\hline$N$ & $E(d d \mu)$ & $E(t t \mu)$ & $N$ & $E(d t \mu)$ \\
\hline 3000 & -0.4887083323489 & -0.5125686534551 & 3300 & -0.50011808395873 \\
\hline 3200 & -0.4887083323559 & -0.5125686535939 & 3500 & -0.50011808395891 \\
\hline 3400 & -0.4887083323604 & -0.5125686537061 & 3700 & -0.50011808395906 \\
\hline 3600 & -0.4887083323625 & -0.5125686537938 & 3840 & -0.50011808395915 \\
\hline 3800 & -0.4887083323638 & -0.5125686538630 & 4000 & -0.50011808395921 \\
\hline$A$ & $-0.488708332375(10)$ & $-0.51256865410(11)$ & $A$ & $-0.50011808395935(5)$ \\
\hline$E^{a}$ & $-0.4887083323645[2]$ & $-0.5125686539435[2]$ & $E^{a}$ & $\begin{array}{lllll}-0.500 & 118 & 083 & 959 & 201\end{array}$ \\
\hline$E^{a}$ & -0.48870833128 [17] & -0.500 11808390 [17] & $E^{a}$ & $-0.51256865199[17]$ \\
\hline
\end{tabular}

${ }^{(a)}$ The best variational energies known from earlier calculations. 
TABLE VI: The total variational energies in muon atomic units for all 22 bound states in the six muonic molecular ions.

\begin{tabular}{lcc}
\hline \hline ion & $S(L=0)$-state & $S^{*}(L=0)$-state \\
\hline$p p \mu-0.49438682024893480876325(10)$ & - \\
$p d \mu$ & $-0.51271179650151455(1)$ & - \\
$p t \mu$ & $-0.5198800897829210(5)$ & - \\
$d d \mu$ & $-0.531111135402386455(2)$ & $-0.4797063803689049978(2)$ \\
$d t \mu$ & $-0.5385949750614133(2)$ & $-0.48806535785170680(5)$ \\
$t t \mu$ & $-0.54637422561381672890(3)$ & $-0.496762894249561327(1)$ \\
\hline ion & $P(L=1)-$ state & $P^{*}(L=1)-$ state \\
\hline$p p \mu$ & $-0.46845843630338535(5)$ & - \\
$p d \mu$ & $-0.490664169479327(1)$ & - \\
$p t \mu$ & $-0.499492029991539(1)$ & - \\
$d d \mu$ & $-0.51362395679268195(2)$ & $-0.4736867338427270(5)$ \\
$d t \mu$ & $-0.523191456315960(1)$ & $-0.48199152997385(5)$ \\
$t t \mu$ & $-0.53326344982038328(2)$ & $-0.48990866750494330(8)$ \\
\hline ion & $D(L=2)-$ state & - \\
\hline$d d \mu$ & $-0.488708332375(10)$ & - \\
$d d \mu$ & $-0.50011808395935(5)$ & - \\
\hline \hline
\end{tabular}

TABLE VII: The convergence of the $\left\langle r_{21}\right\rangle,\left\langle r_{31}^{2}\right\rangle,\left\langle\delta_{21}\right\rangle$ and $\nu_{31}$ expectation values for the ground (bound) $S(L=0)$-states of some muonic molecular ions (in muon-atomic units).

\begin{tabular}{lllll}
\hline \hline \multicolumn{2}{c}{$p p \mu$} & $d t \mu$ & $p d \mu$ & $d d \mu$ \\
\hline$N$ & $\left\langle r_{21}\right\rangle$ & $\left\langle r_{31}^{2}\right\rangle$ & $\left\langle\delta_{32}\right\rangle$ & $\nu_{31}$ \\
\hline 3300 & 3.299486184357381476285 & 5.8818538929815795 & 0.173456203086 & -0.9466714285 \\
3500 & 3.299486184357381476271 & 5.8818538929815783 & 0.173456202965 & -0.9466714315 \\
3700 & 3.299486184357381476272 & 5.8818538929815769 & 0.173456202754 & -0.9466714323 \\
3840 & 3.299486184357381476267 & 5.8818538929815760 & 0.173456202768 & -0.9466714337 \\
\hline A & $3.299486184357381476260(20)$ & $5.8818538929815755(15)$ & $0.173456202768(15)$ & $-0.9466714337(14)$ \\
\hline \hline
\end{tabular}


TABLE VIII: The bound state properties $X$ computed for the ground $S(L=0)$-state and excited $S^{*}(L=0)$-state in the $p d \mu$ and $d t \mu$ muonic molecular ions (in muon-atomic units).

\begin{tabular}{|c|c|c|c|}
\hline$X$ & $p d \mu(S(L=0)$-state & $d t \mu(S(L=0)-$ state & $d t \mu\left(S^{*}(L=0)\right.$-state \\
\hline$\left\langle r_{31}^{-1}\right\rangle$ & $0.6411463715926726(1)$ & $0.7227000085308685(3)$ & $0.5146887540974253(3)$ \\
\hline$\left\langle r_{32}^{-1}\right\rangle$ & $0.7533736132562925(1)$ & $0.7583156106645547(3)$ & $0.7053752991037914(3)$ \\
\hline$\left\langle r_{21}^{-1}\right\rangle$ & $0.3690963918459403(3)$ & $0.4038256690725974(5)$ & $0.2439333374978041(6)$ \\
\hline$\left\langle r_{31}\right\rangle$ & $2.451487588757093(2)$ & $2.117912246542931(3)$ & $3.933235704904257(3)$ \\
\hline$\left\langle r_{32}\right\rangle$ & $2.087699148755371(2)$ & $2.023720495653520(3)$ & $2.738751041467644(3)$ \\
\hline$\left\langle r_{21}\right\rangle$ & $3.100710403484118(3)$ & $2.747914131666301(5)$ & $5.161228965846044(5)$ \\
\hline$\left\langle r_{31}^{2}\right\rangle$ & $8.033494173462087(3)$ & $5.881853892981577(4)$ & $22.39719316098318(5)$ \\
\hline$\left\langle r_{21}^{2}\right\rangle$ & $11.082902111235402(4)$ & $8.2873253005241016(6)$ & $30.631300336084518(6)$ \\
\hline$\left\langle r_{32}^{3}\right\rangle$ & $20.6547093169798(2)$ & $17.4696964524501(3)$ & $65.2551239488477(3)$ \\
\hline$\left\langle r_{21}^{3}\right\rangle$ & $42.147963983631(4)$ & $27.208343544797(5)$ & $201.45178140015(6)$ \\
\hline$\left\langle-\frac{1}{2} \nabla_{1}^{2}\right\rangle$ & $0.2806191952124375(7)$ & $0.3910764762722844(9)$ & $0.3836327499261653(9)$ \\
\hline$\left\langle-\frac{1}{2} \nabla_{2}^{2}\right\rangle$ & $0.3674609039968256(7)$ & $0.4218837824381256(9)$ & $0.4476331914219544(9)$ \\
\hline$\left\langle-\frac{1}{2} \nabla_{3}^{2}\right\rangle$ & $0.46041133628342744(4)$ & $0.50069529865293120(5)$ & $0.50035761171633245(6)$ \\
\hline$\left\langle\delta\left(\mathbf{r}_{31}\right)\right\rangle$ & $0.11770973301(5)$ & $0.15452554329(7)$ & $0.10723299252(7)$ \\
\hline$\nu_{31}$ & $-0.898787990(3)$ & $-0.946671420(5)$ & $-0.946671472(5)$ \\
\hline$\nu_{31}^{a}$ & -0.8987879287819516 & -0.9466714310522288 & -0.9466714310522288 \\
\hline$\left\langle\delta\left(\mathbf{r}_{32}\right)\right\rangle$ & $0.17345620275(5)$ & $0.17451466503(7)$ & $0.17875743059(7)$ \\
\hline$\nu_{32}$ & $-0.9466716379(8)$ & $-0.9637483436(10)$ & $-0.9637483713(10)$ \\
\hline$\nu_{32}^{a}$ & -0.9466714310522287 & -0.9637483334950246 & -0.9637483334950246 \\
\hline$\left\langle\delta\left(\mathbf{r}_{21}\right)\right\rangle$ & $1.4616938(4) \cdot 10^{-5}$ & $8.870847(1) \cdot 10^{-7}$ & $7.416512(1) \cdot 10^{-7}$ \\
\hline$\nu_{21}$ & $5.9192044(3)$ & $10.644434(5)$ & $10.644863(5)$ \\
\hline$\nu_{21}^{a}$ & 5.9191833130515522 & 10.644186704849556 & 10.644186704849556 \\
\hline$\left\langle\delta_{321}\right\rangle$ & $2.28129(2) \cdot 10^{-5}$ & $1.61921(3) \cdot 10^{-6}$ & $1.35816(3) \cdot 10^{-6}$ \\
\hline
\end{tabular}

${ }^{(a)}$ The exact value. 
TABLE IX: Calculations of the mass gradients for the $P(L=1)$-states in the $p p \mu, d d \mu$ and $t t \mu$ muonic molecular ions in muon-atomic units where $\hbar=1, e=1$ and $m_{\mu}=206.768262 m_{e}=1$. The notations used in this Table correspond to the notations used in Eq.(6).

\begin{tabular}{|c|c|c|}
\hline & $p p \mu$ & $d d \mu$ \\
\hline$E\left(m_{\mu}-2 h\right)$ & -0.468453822026274128170 & -0.513619514847688534232 \\
\hline$E\left(m_{\mu}-h\right)$ & -0.468456129164644884357 & -0.51362 1714727907740353 \\
\hline$E\left(m_{\mu}+h\right)$ & -0.4684607434 42495278996 & -0.513626177762223263542 \\
\hline$E\left(m_{\mu}+2 h\right)$ & -0.468463050581974916597 & -0.51362 8398729794443654 \\
\hline \multirow[t]{2}{*}{$\alpha$} & $-2.307138925197(10) \cdot 10^{-3}$ & $-2.235032701523(10) \cdot 10^{-3}$ \\
\hline & $t t \mu$ & $(t t \mu)^{*}$ \\
\hline$E\left(m_{\mu}-2 h\right)$ & -0.5332587089793171418 & -0.4899043312785580219 \\
\hline$E\left(m_{\mu}-h\right)$ & -0.5332610794006558570 & -0.4899064993922363403 \\
\hline$E\left(m_{\mu}+h\right)$ & -0.53326 58202384992057 & -0.4899108356167417247 \\
\hline$E\left(m_{\mu}+2 h\right)$ & -0.5332681906550038459 & -0.4899130037274395589 \\
\hline$\alpha$ & $-2.370418921674(11) \cdot 10^{-4}$ & $-2.168112263462(11) \cdot 10^{-4}$ \\
\hline
\end{tabular}


TABLE X: The total and binding energies of a weakly bound $P^{*}(L=1)$-state in the $d d \mu$ muonic molecular ion as the function of the muon mass $\tilde{m}_{\mu}=m_{\mu}+y$, where $m_{\mu}=206.768262 m_{e}$ and $y$ is a non-negative real number. All energies have been recalculated to muon-atomic units where $\hbar=1, e=1$ and $m_{\mu}=206.768262 m_{e}=1$. The binding energies $\varepsilon$ are given in electronoVolts $(\mathrm{eV})$. $N$ is the total number of basis functions used in variational expansion.

\begin{tabular}{ccc}
\hline \hline$y$ & $E(N=3300)$ & $\varepsilon(N=3300)$ \\
\hline 0 & -0.4736867338427258104 & -1.97498808799965 \\
1 & -0.4712335469779210548 & -1.68187156159084 \\
2 & -0.4688058829862271553 & -1.39988399201543 \\
3 & -0.4664034583422745329 & -1.12949080447425 \\
4 & -0.4640260218725323738 & -0.87131211085906 \\
5 & -0.4616733706509872075 & -0.62621871045415 \\
6 & -0.4593453849322646260 & -0.39554090739252 \\
7 & -0.4570421278654125672 & -0.18165985634448 \\
7.9 & -0.4549909488076416492 & -0.00875602540622 \\
7.95 & -0.4548776498295269995 & -0.00002551843452 \\
8 & -0.4547644307696878137 & +0.00854711729854 \\
\hline$y$ & $E(N=3840)$ & $\varepsilon(N=3840)$ \\
\hline 7.95 & -0.4548776454620138526 & -0.00007865184962 \\
\hline \hline
\end{tabular}


TABLE XI: The total and binding energies of a weakly bound $P^{*}(L=1)$-state in the $d t \mu$ muonic molecular ion as the function of the muon mass $\tilde{m}_{\mu}=m_{\mu}+y$, where $m_{\mu}=206.768262 m_{e}$ and $y$ is a non-negative real number. All energies have been recalculated to muon-atomic units where $\hbar=1, e=1$ and $m_{\mu}=206.768262 m_{e}=1$. The binding energies $\varepsilon$ are given in electronoVolts $(\mathrm{eV})$. $N$ is the total number of basis functions used in variational expansion.

\begin{tabular}{lll}
\hline \hline$y$ & $E(N=3700)$ & $\varepsilon(N=3700)$ \\
\hline 0.0 & -0.48199152997371597 & -0.66033868534010 \\
1.0 & -0.48184465428112405 & -0.31071047997243 \\
1.8 & -0.48173196328443396 & -0.05573790640587 \\
1.9 & -0.48171831993605128 & -0.02623616728477 \\
1.95 & -0.48171161233515334 & -0.01212193523051 \\
1.98 & -0.48170758889627615 & -0.00365631831137 \\
2.0 & -0.48170482239836190 & +0.00246722246561 \\
\hline$y$ & $E(N=3840)$ & $\varepsilon(N=3840)$ \\
\hline 1.95 & -0.48171161309408528 & -0.01212624559609 \\
1.98 & -0.48170759005686002 & -0.00366291081502 \\
2.0 & -0.48170492020870050 & +0.00191157404752 \\
\hline \hline
\end{tabular}

